Peter Ellguth, Susanne Kohaut, Iris Möller*

\title{
Wo schaffen es Frauen an die Spitze? Eine empirische Analyse mit Betriebsdaten**
}

\section{Zusammenfassung}

Der Beitrag geht der Frage nach, welche betrieblichen Faktoren die Präsenz von Frauen in Führungspositionen beeinflussen. Hierbei interessiert uns auch die Rolle des Betriebsrats. Für unsere multivariate Analyse nutzen wir das IAB-Betriebspanel 2014 und verwenden Zähldatenmodelle mit der Anzahl Frauen im Führungsteam als abhängiger Variablen. Wir schätzen sowohl Modelle für die erste als auch für die zweite Führungsebene. Für die erste Führungsebene lassen sich Hinweise auf die in der Literatur beschriebenen Barrieren finden, die als gläserne Klippen und Böden bzw. Etagen, auf denen Frauen kleben bleiben, umschrieben werden. Insgesamt vermitteln die Ergebnisse aber vor allem den Eindruck, dass Frauen in ,unattraktiven“ Betrieben eher in Führungspositionen zu finden sind.

\section{Where do women reach top positions? An empirical analysis with establishment data}

\section{Abstract}

This study investigates the determinants of women in top management positions, especially the role of works councils. For our multivariate analysis we use data of the IAB Establishment Panel 2014 and apply count data models as the dependent variable is the number of women in top positions. We provide separate estimations for the first and the second management level. Our findings confirm for the top executive level factors described in the literature as "glass cliffs" and "sticky floors". All in all the results indicate that women reach leading positions especially in establishments, which are "less attractive".

Key words: Executive Position; Gender; Equality; Works Councils; Führungspositionen; Gender; Chancengleichheit, Betriebsrat (JEL: J16, J53, J71, M12, M51)

* Peter Ellguth, Institut für Arbeitsmarkt- und Berufsforschung der Bundesagentur für Arbeit, Regensburger Str. 100, D-90478 Nürnberg. E-Mail: peter.ellguth@iab.de

Dr. Susanne Kohaut, Institut für Arbeitsmarkt- und Berufsforschung der Bundesagentur für Arbeit, Regensburger Str. 100, D-90478 Nürnberg. E-Mail: susanne.kohaut@iab.de

Dr. Iris Möller, Institut für Arbeitsmarkt- und Berufsforschung der Bundesagentur für Arbeit, Regensburger Str. 100, D-90478 Nürnberg. E-Mail: iris.moeller@iab.de

** Artikel eingegangen: 28.04.2016, revidierte Fassung akzeptiert nach doppelt-blindem Begutachtungsverfahren: 08.01.2017. 


\section{Einleitung}

Die politische Debatte über die Repräsentanz von Frauen in Führungspositionen deutscher Unternehmen wird unter verschiedenen Aspekten geführt. An erster Stelle steht natürlich die Diskussion um Chancengleichheit und Partizipation. Schon aus Gründen der Gerechtigkeit sollten Frauen vor dem Hintergrund vergleichbarer Bildungsniveaus entsprechend ihrem Anteil an den Beschäftigten in leitenden Positionen vertreten sein. Ein weiterer Diskussionsstrang betrifft Diversity-Aspekte. Verschiedene Studien weisen auf die positive Wirkung von Geschlechterdiversität in Führungsteams auf den Unternehmenserfolg hin. Die vorliegende Literatur zeigt allerdings, dass dieser Zusammenhang komplex und stark kontextabhängig ist (für Deutschland z.B. Joecks, Pull, \& Vetter, 2012 oder Jirjahn, 2011). Nicht zuletzt wird die Diskussion über Frauen in Führungspositionen auch im Rahmen der Debatte um Fachkräftebedarf und demographischen Wandel geführt. Hierbei wird die verstärkte Rekrutierung von Frauen als Mittel zur Verbesserung und Erweiterung des Talentpools gesehen (z.B. Die bayerischen Metall- und Elektro-Arbeitgeber, 2016). Insgesamt besteht ein mehr oder weniger breiter politischer Konsens, dass in deutschen Betrieben Frauen so in Führungspositionen vertreten sein sollten, wie es ihrem Anteil an den Beschäftigten entspricht. ${ }^{1}$ Die Realität sieht jedoch anders aus: Die vorliegenden Daten beschreiben ein Bild der deutschen Wirtschaft, das noch ein beträchtliches Stück von dieser Vorstellung entfernt ist.

Nicht zuletzt aufgrund der stagnierenden Zahlen wurde zum 1. Mai 2015 nach einer jahrelangen teils sehr kontrovers geführten Diskussion ein Gesetz für die gleichberechtigte Teilhabe von Frauen und Männern an Führungspositionen in der Privatwirtschaft und im öffentlichen Dienst beschlossen. Mit dem im Januar 2016 in Kraft getretenen Gesetz soll mittelfristig der Anteil von Frauen in Führungspositionen verbessert und letztlich Chancengleichheit von Frauen und Männern erreicht werden. Das Gesetz schreibt eine verbindliche Frauenquote von 30 Prozent in Aufsichtsräten von börsennotierten und paritätisch mitbestimmten Unternehmen fest. Zudem müssen diese Unternehmen ebenso wie Aktiengesellschaften, Kommanditgesellschaften auf Aktien, GmbHs, eingetragene Genossenschaften und Versicherungsvereine mit mehr als 500 Arbeitnehmern Zielgrößen zur Erhöhung des Frauenanteils auch in Vorständen und der obersten Managementebene festlegen und darüber öffentlich berichten.

Inwieweit das Gesetz die intendierte Wirkung entfaltet, kann mit den im Moment zur Verfügung stehenden Daten noch nicht überprüft werden. Zahlen aus dem IAB-Betriebspanel zur Situation in der deutschen Privatwirtschaft 2014 zeigen, dass nur ein Viertel der Toppositionen mit Frauen besetzt sind - ohne jeglichen Fortschritt in den letzten zehn Jahren (Kohaut \& Möller, 2016). Auf der zweiten Führungsebene stieg im gleichen Zeitraum der Frauenanteil an, aber auch dieser konnte nicht den Anteil der Frauen an der Gesamtbeschäftigung erreichen. Auch andere Studien kommen zu vergleichbaren Ergebnissen (Holst \& Kirsch, 2016). Vor allem auf der ersten Führungsebene besteht - nach wie vor - eine beträchtliche Lücke, die Grund genug liefert, sich mit den betrieblichen Umständen dieses Missverhältnisses zu beschäftigen.

Übereinstimmung herrscht darüber, dass die Unterrepräsentation von Frauen in Führungspositionen vielfältige Ursachen hat (z.B. Orser \& Leck, 2010). Dabei lassen sich grundsätzlich Erklärungen, die sich auf die Angebotsseite des Arbeitsmarktes beziehen, von nach-

1 Die Differenz zwischen dem Anteil weiblicher Beschäftigter und ihrem Anteil auf der obersten Führungsebene wird auch als Gender Leadership Gap bezeichnet (Holst \& Friedrich, 2016). 
frageseitigen Faktoren unterscheiden (Graham, Belliveau, \& Hotchkiss, 2016). Bei ersteren geht es vor allem um geschlechtsspezifische Persönlichkeitseigenschaften, Ausbildungsentscheidungen und Präferenzen bei der Berufswahl, die Karrierechancen beeinflussen. Hinzu kommen traditionell arbeitsteilige Strukturen in den Familien, die vermittelt über familienbedingte Ausfallzeiten und eingeschränkte berufliche „Verfügbarkeiten“ die Chancen für Frauen, in eine Führungsposition zu gelangen, verringern.

Unser Interesse gilt allerdings der Nachfrageseite des Arbeitsmarktes. Dabei stehen Strukturen und Praktiken in Betrieben im Fokus, die möglicherweise Karrierewege beeinflussen. So kann eine über lange Jahre gewachsene betriebliche Kultur, in der auch Geschlechtsstereotypen verankert sind, den Aufstieg von Frauen in Top-Positionen erschweren oder sogar verhindern. Ein Beispiel hierfür wären Auswahlprozesse, die weder standardisiert noch transparent sind und in denen die Rekrutierung des Führungspersonals durch persönliche Kontakte und männerdominierte informelle Netzwerke geschieht.

Umgekehrt kann die Organisationskultur so gestaltet werden, dass das Geschlechterverhältnis im Management positiv beeinflusst wird, indem - als Reaktion auf die zunehmend als gesellschaftlich nicht akzeptabel empfundene Benachteiligung von Frauen - beispielsweise spezielle Maßnahmen und Programme zur Förderung von Frauen angeboten werden.

Der Betriebsrat als Institution, der die Arbeitsbedingungen in vielfacher Weise beeinflusst und wesentlicher Bestandteil der Betriebskultur ist, sollte hier mit ins Kalkül gezogen werden. Betriebsratsgremien sind nach wie vor von Männern dominiert. Das gilt insbesondere für die Position des Vorsitzes (Stettes, 2015). Insofern liegt es nahe, Betriebsräte als Teil einer männlich dominierten Organisationskultur zu betrachten, deren Interesse eher nicht der Förderung von Frauen gilt. Auf der anderen Seite kommt dem Betriebsrat laut Betriebsverfassungsgesetz die allgemeine Aufgabe zu, Chancengleichheit zu fördern und Diskriminierung zu verhindern $(\S \S 75,80)$. Insofern ist es eine offene Frage, welche Bedeutung der Betriebsrat für die Präsenz von Frauen in Führungspositionen hat.

Vor diesem Hintergrund wollen wir empirisch überprüfen, welche betrieblichen Faktoren sich identifizieren lassen, die die Präsenz von Frauen in Führungspositionen beeinflussen. Hierbei interessiert uns neben dem Betriebsrat insbesondere auch, ob Frauen in Betrieben mit den angesprochenen personalpolitischen Maßnahmen tatsächlich häufiger auf den oberen Führungsebenen zu finden sind. Für unsere multivariate Analyse nutzen wir das IAB-Betriebspanel und verwenden Zähldatenmodelle mit der Anzahl Frauen im Führungsteam als abhängiger Variablen. Wir schätzen sowohl Modelle für die erste als auch für die zweite Führungsebene. Wir verbinden mit unserer Betrachtung keinerlei kausalen Erklärungsanspruch. Unser Anliegen lässt sich eher als multivariate Identifikation relevanter betrieblicher Charakteristika bezeichnen.

Der Aufsatz ist wie folgt gegliedert: Zunächst wird der Stand der Forschung dargestellt. Der Abschnitt „Empirische Überprüfung“ beginnt mit einer kurzen Beschreibung des IAB-Betriebspanels und einem deskriptiven Überblick. Es schließt sich die Operationalisierung der Variablen und eine Darstellung der verwendeten Schätzmethoden an. Der empirische Teil schließt mit der Interpretation der Schätzergebnisse. Den Abschluss des Aufsatzes bildet das Fazit. 


\section{Hintergrund und Stand der Forschung}

In der Literatur werden verschiedene Ursachen für die Unterrepräsentation von Frauen in Führungspositionen diskutiert. Nicht nur die individuellen Präferenzen und Fähigkeiten der Frauen (individuelle Ebene) und die Bedingungen im Unternehmen (Unternehmensebene) spielen hierbei eine wesentliche Rolle. Auch Staat und Gesellschaft (Makroebene) haben über die Gesetzgebung und gesellschaftliche Rollenbilder einen wesentlichen Einfluss auf die Karriere von Frauen (Krishnan, 2009). Dementsprechend unterscheiden sich die Perspektiven, mit der die vielen theoretischen und empirischen Arbeiten an dieses Thema herangehen (für einen Überblick siehe z.B. Calas, Smircich, \& Holvino, 2014 oder Smith \& Verner, 2013). In dem vorliegenden Papier interessieren uns die Determinanten der Präsenz von Frauen in Führungspositionen, also die Frage, in welchen Betrieben es Frauen in Führungspositionen schaffen. Damit nimmt natürlich die Unternehmens- oder Betriebsebene eine zentrale Stellung ein. Nichtsdestoweniger soll auch ein kurzer Überblick über die individuellen Erklärungsmuster und die Makroebene gegeben werden, auch weil die unterschiedlichen Ebenen z.T. mit einander verzahnt und damit nicht immer trennscharf sind.

Studien, die die individuelle Ebene im Blick haben, suchen die Gründe für die Unterrepräsentanz von Frauen in Führungspositionen in den unterschiedlichen Charakteristika von Männern und Frauen. Die Human-Kapital-Theorie geht davon aus, dass eine bestimmte Humankapitalausstattung, also z.B. Ausbildung und Berufserfahrung, notwendig ist, um in eine bestimmte Position zu gelangen. Die Gründe für die geringe Präsenz von Frauen in Führungspositionen werden in dieser inzwischen eher als veraltet betrachteten Theorie folglich bei den Frauen selbst verortet (Busch \& Holst, 2010). Diese Ansätze beschäftigen sich mit den vermeintlichen Defiziten der Frauen, denen bestimmte Eigenschaften und Fähigkeiten abgesprochen werden, die für eine Managementposition als unerlässlich gelten (Holst, 2009; Powell, 2014). Es sind die traditionell als maskulin wahrgenommenen Eigenschaften wie Risikofreude oder Wettbewerbsorientierung, die als Voraussetzung für eine Führungskraft angesehen werden. Obwohl Frauen inzwischen gleichwertige oder sogar höhere Bildungsabschlüsse haben und vermeintlich typisch weibliche Eigenschaften, wie Einfühlungsvermögen, mittlerweile als besonders wichtig für Führungskräfte gelten, schaffen sie es dennoch deutlich seltener in Toppositionen. Dabei spielen Rollenzuweisungen und Geschlechterstereotype nach wie vor sicherlich eine wichtige Rolle und stellen ein wesentliches Hindernis für Frauen dar. Diese Stereotype beeinflussen aber auch die Frauen selbst und verhindern, dass sie mit dem gleichen Selbstverständnis wie Männer ihre Karriere planen.

Hinzu kommt, dass in Deutschland Frauen nach wie vor die Hauptverantwortung bei der Kindererziehung und im Haushalt tragen. Familienbedingte Unterbrechungen und Teilzeitarbeit werden deshalb sehr viel häufiger von Frauen in Anspruch genommen. Die Ausfallzeiten während der Familienbildungsphasen verfestigen zusätzlich die damit einhergehenden traditionellen Strukturen in den Familien und fallen zeitlich meist mit der wichtigen Phase der Karrierebildung zusammen. Die Chancen für Frauen auf eine Führungsposition verringern sich auch (Holst, 2009; Schulz \& Blossfeld, 2006), da familienbedingte Ausfallzeiten meist nicht mehr aufgeholt werden können.

Ein weiteres gesellschaftliches Phänomen (Makroebene) in Deutschland ist die nach wie vor ausgeprägte horizontale Segregation der Geschlechter im Erwerbsbereich. Sie ist entscheidend geprägt durch die unterschiedliche Berufswahl von Frauen und Männern, die sich auf ganz verschiedene Berufsfelder konzentrieren (Allmendinger \& Hinz, 1999) und 
für ungleiche Aufstiegs- und Verdienstmöglichkeiten mitverantwortlich sind. So ergreifen Frauen häufiger als Männer Berufe mit strukturellen Nachteilen, die weniger Möglichkeiten bieten, in eine Führungsposition zu gelangen (Holst \& Wiemer, 2010). Zusammen mit der dadurch entstehenden vertikalen Segregation in statushohe und statusniedrige Tätigkeiten in einzelnen Berufsfeldern ,trägt die berufliche Segregation nach Geschlecht stark zu Einkommens- und Machtunterschieden zwischen Frauen und Männern bei“ (Allmendinger \& Hinz, 1999, S. 191). Frauen arbeiten jedoch nicht nur in statusniedrigen Berufsfeldern bzw. Branchen, sondern auch häufiger in eher kleinen Betrieben, die im Allgemeinen niedrigere Löhne zahlen als große. Da Frauen eher Führungspositionen in diesen kleineren Betrieben innehaben, üben sie auch deutlich weniger Macht aus als Männer (Busch \& Holst, 2009; Holst \& Friedrich, 2016).

Die Forschung zu genderspezifischen Karrierewegen und der Diskriminierung von Frauen beleuchtet inzwischen auch den Einfluss von Organisationen auf die Karriere von Frauen und damit die Unternehmensebene. Es werden zunehmend organisationsinterne Faktoren in Betracht gezogen. Hierbei geht es vor allem darum, die Organisationskultur, also die Rolle, die betriebliche Traditionen und nicht hinterfragte Praktiken und Gepflogenheiten spielen, mit zu berücksichtigen.

Am bekannteste dürfte der Glass-Ceiling-Ansatz sein, in dem die Unterrepräsentanz von Frauen in Managementpositionen beschrieben wird (Cotter, Hermsen, Ovadia, \& Vanneman, 2001). Eine Vielzahl von Arbeiten hat sich mit der Frage beschäftigt, ob eine solche ,gläserne Decke“ wirklich beobachtet werden kann. Man geht davon aus, dass sich die geringe Repräsentanz von Frauen in Führungspositionen nicht allein durch individuelle Charakteristika, wie Schulabschluss oder Berufsausbildung, der weiblichen Beschäftigten erklären lässt, sondern ,unsichtbare“ Barrieren und festgefügte Mechanismen den Frauen den Zugang zu Führungspositionen versperren. Frauen müssen demnach Hindernisse überwinden, die Männern nicht im Weg stehen. Wie diese beobachtbaren Glass-Ceiling-Effekte letztlich erklärt werden können und worin sie ihren Ursprung haben, darüber gibt es in der Literatur keine Einigkeit, ebenso wenig wie bei der Frage, wie dieser Effekt am besten zu operationalisieren ist (für einen Überblick siehe Jackson \& O'Callaghan, 2009).

Zwar hat sich in den letzten Jahren der Anteil von Frauen auf den unteren Führungsebenen deutlich erhöht, trotzdem schaffen Frauen nur selten den Aufstieg in Toppositionen. Für sie erweisen sich die unteren Führungsebenen häufig als „sticky floors“ (Booth, Francesconi, \& Frank, 2003). Erklärt wird dies mit der fehlenden Flexibilität von Frauen im Vergleich zu Männern aufgrund ihrer familiären Verpflichtungen, die es ihnen erschweren, attraktive Angebote anderer Arbeitgeber anzunehmen (Smith N., Smith V., \& Verner 2013). Letzteres wird auch als Erklärung für die nicht unerheblichen Gehaltsunterschiede zwischen weiblichen und männlichen Führungskräften herangezogen. Nach wie vor verdienen Frauen, auch in Toppositionen, weniger als ihre männlichen Kollegen (Smith et al., 2013; Fietze, Holst, \& Tobsch, 2011).

In jüngerer Zeit wurden die Ansätze um die ,glass cliff“ Hypothese erweitert. Verschiedene empirische Studien zeigen, dass Frauen eher in Betrieben mit wirtschaftlichen Schwierigkeiten in Führungspositionen gelangen (Billing \& Alvesson, 2014; Metz \& Kulik, 2014). Auf den ersten Blick könnte dies als Hinweis auf die besonderen Managementfähigkeiten von Frauen interpretiert werden, allerdings ist die Ausübung einer Führungsposition in einer wirtschaftlich schwierigen Situation mit einem sehr viel höheren Risiko zu scheitern verbun- 
den. Ein möglicher Misserfolg wird dann jedoch als ein Beleg für die mangelnde Befähigung weiblicher Führungskräfte gesehen (Barretto \& Ellemers, 2005).

Eine Reihe von Forschungsarbeiten hat in den letzten Jahren eine weitere wichtige Barriere für die Aufstiegschancen von Frauen aufgezeigt: Überlange Arbeitszeiten und eine Präsenzkultur hält Frauen eher von den Chefetagen fern, da sie zumindest in Deutschland nach wie vor die Hauptverantwortung für die Familie tragen und deshalb ihre Zeit nicht ausschließlich der Arbeit widmen können (Billing \& Alvesson, 2014).

Um eingefahrene betriebliche Strukturen aufzubrechen und Aufstiegsbarrieren für Frauen abzubauen, wird eine breite Palette betrieblicher Maßnahmen diskutiert (für einen Überblick: Metz \& Kulik, 2014, S. 188). Dazu zählen ganz unterschiedliche Vorschläge wie Workshops, die sich speziell mit Themen der Geschlechtergerechtigkeit befassen, die Festlegung von klaren Zielen bei der Besetzung von Führungspositionen und deren regelmäßige Überprüfung ebenso wie die Abschaffung langer Anwesenheitszeiten. Auch flexible Arbeitszeitmodelle und personalpolitische Maßnahmen zur gezielten Förderung von Frauen wie Frauenförderpläne oder spezielle Weiterbildungsangebote werden in diesem Zusammenhang diskutiert.

Geschlechtsspezifische Stereotypen können auch innerhalb von Organisationen eine wesentliche Rolle spielen und werden am besten mit dem Begriff ,think manager - think male" zusammengefasst (Powell, 2014, S. 254f.). Diese Stereotypen behindern - so sie nicht bewusst hinterfragt und aufgebrochen werden - Frauen nach wie vor in ihrer Karriere. Die meisten Betriebe sind zumindest auf der Führungsebene männerdominiert. Dominante Gruppen neigen jedoch dazu, wiederum Mitglieder der eigenen Gruppen auszuwählen oder wie es Metz und Kulik (2014, S. 186) formulieren: ,... women are likely to continue to encounter group membership obstacles as members of the (female) out-group in current organizations ${ }^{\text {c }}$ prodominantly male hierarchies.“

Eine solche Organisationskultur wird jedoch nicht nur von männlichen Führungskräften geprägt, sondern möglicherweise auch von den Betriebsräten. Folglich stellt sich die Frage, welche Rolle der Betriebsrat bei der Besetzung von Führungspositionen spielt. Theoretische Ansätze hierzu sucht man vergebens. Das mag vielleicht am institutionellen Hintergrund liegen, der einen Einfluss des Betriebsrats auf die geschlechtsspezifische Zusammensetzung der Leitungsebene nicht unmittelbar nahe legt.

Das Betriebsverfassungsgesetz garantiert dem Betriebsrat zwar ein abgestuftes Arsenal an Mitwirkungsrechten vor allem in sozialen Angelegenheiten. Seine Rechte bei Einstellungen sind aber nicht sehr ausgeprägt und gerade in Angelegenheiten, die das Führungspersonal in leitenden Positionen (leitende Angestellte) betreffen, hat er so gut wie keine Mitspracherechte. Aber seit der Reform der Betriebsverfassungsgesetzes 2001 hat der Betriebsrat die Aufgabe, Chancengleichheit zu fördern und Diskriminierung zu verhindern (§§ 75, 80) und damit einen rechtlichen Ansatzpunkt, hier aktiv zu werden. Er könnte sich beispielsweise um diskriminierungsfreie Regelungen bei der Ausschreibung und der Besetzung von Stellen bemühen, die dann auch für Führungspositionen angewendet werden oder entsprechende Verhaltenskodizes mitgestalten. Insgesamt betrachtet könnte es sich der Betriebsrat also durchaus zu seiner Aufgabe machen, auf die (geschlechtsspezifische) Besetzung von Führungspositionen einzuwirken und hätte dazu auch die Möglichkeiten.

Auf der anderen Seite gelten Betriebsratsgremien immer noch als Bastion der Männer. Seit der Reform des Betriebsverfassungsgesetzes 2001 muss zwar das Geschlecht, dass in der Belegschaft in der Minderheit ist, proportional im Betriebsrat vertreten sein ( $\$ 15$ Abs. 2 BetrVG auch zu den Ausnahmeregelungen). Für die Besetzung des Betriebsratsvorsitzes 
gibt es aber keine geschlechtssensible Regelung. Die aus Erhebungen nach den letzten Betriebsratswahlen zur Verfügung stehenden Zahlen gehen auseinander, dokumentieren aber alle eine deutliche Unterrepräsentierung der Frauen. Der DGB kommt in seinem Trendreport Wahlen 2014 auf einen Frauenanteil beim Vorsitz von 29,1 Prozent (Greifenstein, Kißler, \& Lange, 2014). Die IW-Betriebsratswahlbefragung 2014 spricht von nur 16 Prozent Frauen als Betriebsratsvorsitzende (Stettes, 2015, S. 17). Die neusten Daten stammen aus der WSIBetriebsrätebefragung 2015: Hiernach beträgt der Frauenanteil 26,9 Prozent (Baumann \& Brehmer, 2016). Hinzu kommt nach den dort erhobenen Zahlen zum Alter des/der Betriebsratsvorsitzenden eine im Vergleich zum Rest der Beschäftigten deutlich nach oben verschobenen Altersstruktur, mit über 60 Prozent der Vorsitzenden in der Altersgruppe ab 51 Jahren. Diese Argumente sprechen wohl eher dafür, Betriebsräte als Teil einer männlich dominierten Organisationskultur zu betrachten, deren Interesse nicht der Förderung von Frauen gilt. In diese Richtung gehen auch Ergebnisse von Klempt \& Klee (2015) in Schätzungen für Baden-Württemberg, die einen eher negativen Zusammenhang zwischen der Existenz eines Betriebsrats und der Präsenz von Frauen in Führungspositionen finden.

\section{Empirische Überprüfung}

\subsection{Datengrundlage}

Das IAB-Betriebspanel ist eine jährliche Befragung von knapp 16.000 Betrieben aller Branchen und Größenklassen in Deutschland. Die Erhebung wird seit 1993 in den westdeutschen und seit 1996 in den ostdeutschen Bundesländern durchgeführt. Ziehungsgrundlage des IAB-Betriebspanels ist die aus der Beschäftigtenstatistik aggregierte Betriebsdatei der Bundesagentur für Arbeit, die alle Betriebe/Dienststellen mit mindestens einem sozialversicherungspflichtig Beschäftigten enthält. Die Betriebe werden nach dem Prinzip der optimalen Schichtung gezogen, wobei die Ziehungswahrscheinlichkeit mit der Betriebsgröße steigt. Als Schichtungsvariablen dienen zehn Betriebsgrößenklassen, 19 Branchen und die Bundesländer. Da es sich um eine Längsschnitterhebung handelt, werden soweit möglich jedes Jahr dieselben Betriebe wieder befragt. Um Neugründungen abzubilden und die Folgen der Panelmortalität auszugleichen, wird die Stichprobe jährlich um zusätzliche Betriebe ergänzt. Zum Ausgleich der Disproportionalität der Zufallsstichprobe werden die deskriptiven Ergebnisse jeweils auf die Eckwerte der Grundgesamtheit laut Betriebsdatei der BA hochgerechnet. Die Erhebung ist repräsentativ für alle Betriebe mit mindestens einem sozialversicherungspflichtigen Beschäftigten. Für weitere Informationen zum IAB-Betriebspanel siehe Fischer, Janik, Müller, \& Schmucker (2008) und Ellguth, Kohaut, \& Möller (2014).

Die Frage zu Frauen in Führungspositionen wurde im IAB-Betriebspanel zwischen 2004 und 2012 im 4-jährigen Rhythmus abgefragt. Mit der Welle 2014 ist der Befragungsabstand auf zwei Jahre verkürzt worden. Erfasst wird die Anzahl der Vorgesetzen auf der obersten und - sofern vorhanden - auf der zweiten Führungsebene und zusätzlich die Anzahl der Frauen auf der jeweiligen Führungsebene. Damit stehen für beide Führungsebenen Informationen zur Präsenz von Frauen zur Verfügung.

Wir begrenzen unser Untersuchungsfeld auf Betriebe der bundesdeutschen Privatwirtschaft, da diese im Fokus des öffentlichen Interesses stehen. Außerdem schließen wir Betriebe mit weniger als fünf Mitarbeitern aus, da eine Berücksichtigung dieser Betriebe, in denen 
die rechtliche Grundlage für die Wahl eines Betriebsrats fehlt, zu systematischen Verzerrungen führen würde.

\subsection{Deskriptive Ergebnisse zu Frauen in Führungspositionen}

Die Zahlen des IAB-Betriebspanels zeigen, dass der Anteil von Frauen auf der obersten Führungsebene mit ca. 25 Prozent seit 2004 nahezu konstant geblieben ist. Auf der zweiten Führungsebene können moderate Zuwächse beobachtet werden (Tabelle 1). Im Jahr 2004 war jede dritte Vorgesetztenposition auf der zweiten Ebene von einer Frau besetzt; zehn Jahre später waren es fast 40 Prozent.

Tab. 1: Frauenanteil auf den ersten beiden Führungsebenen und an allen Beschäftigten*

\begin{tabular}{|c|c|c|c|}
\hline \multicolumn{5}{|c}{ - hochgerechnete Werte - } \\
\hline Anteile in \% & 1. Führungsebene & 2. Führungsebene & alle Beschäftigten \\
\hline 2004 & 24 & 33 & 41 \\
2008 & 25 & 35 & 42 \\
2012 & 26 & 38 & 43 \\
2014 & 25 & 39 & 43 \\
\hline
\end{tabular}

*Betriebe der Privatwirtschaft

Quelle: IAB-Betriebspanel 2004, 2008, 2012, 2014

Jedoch soll die positive Entwicklung zugunsten von Frauen auf der zweiten Führungsebene nicht darüber hinwegtäuschen, dass Frauen nach wie vor in Führungspositionen nicht entsprechend ihres Anteils an der Gesamtbeschäftigung vertreten sind.

Die Unterrepräsentanz von Frauen ist stark von der Betriebsgröße abhängig siehe Tabelle 2. Sowohl der Anteil der Frauen in Führungspositionen, als auch ihr Anteil an der Gesamtbeschäftigung nehmen mit steigender Größenklasse ab. In größeren Betrieben ist die Unterrepräsentanz (bezogen auf den Frauenanteil an der Gesamtbeschäftigung) allerdings höher als in kleineren (diese und weitere Auswertungen siehe Kohaut \& Möller, 2016).

Um einen ersten Eindruck zu gewinnen, werden im Folgenden deskriptive Ergebnisse zur Repräsentanz von Frauen in Führungspositionen in Abhängigkeit der uns insbesondere interessierenden betrieblichen Merkmale präsentiert. Das ist zum einen die Existenz eines Betriebsrats. Wie Tabelle 2 zeigt, gibt es in neun Prozent der Betriebe (der Privatwirtschaft mit 5 und mehr Beschäftigten) eine gesetzlich legitimierte Mitarbeitervertretung. Auch der Verbreitungsgrad des Betriebsrats ist stark abhängig von der Betriebsgröße. In kleinen Betrieben mit bis zu 50 Beschäftigten ist die Existenz eines Betriebsrates mit fünf Prozent eher die Ausnahme, während in Großbetrieben eine solche nahezu selbstverständlich ist; 88 Prozent aller Betriebe der Privatwirtschaft mit mehr als 500 Beschäftigten haben einen Betriebsrat. 
Tab. 2: Anteil der Betriebe mit Betriebsrat und Frauenanteil auf den ersten beiden Führungsebenen

\begin{tabular}{|c|c|c|c|c|c|c|c|}
\hline \multirow{2}{*}{$\begin{array}{l}\text { Anteile in \% } \\
\text { Betriebsgröße* }\end{array}$} & \multirow{2}{*}{$\begin{array}{l}\text { Betriebe } \\
\text { mit BR }\end{array}$} & \multicolumn{3}{|c|}{$\begin{array}{l}\text { Frauenanteil auf der ersten } \\
\text { Führungsebene }\end{array}$} & \multicolumn{3}{|c|}{$\begin{array}{c}\text { Frauenanteil auf der zweiten } \\
\text { Führungsebene }\end{array}$} \\
\hline & & ohne BR & mit BR & Gesamt & ohne BR & mit BR & Gesamt \\
\hline 5 bis 50 Beschäftigte & 5 & 25 & 26 & 26 & 45 & 42 & 45 \\
\hline 51 bis 100 Beschäftigte & 37 & 22 & 18 & 20 & 38 & 29 & 34 \\
\hline 101 bis 199 Beschäftigte & 55 & 24 & 17 & 20 & 36 & 27 & 30 \\
\hline 200 bis 500 Beschäftigte & 72 & 24 & 16 & 18 & 31 & 27 & 28 \\
\hline 501 und mehr Beschäftigte & 88 & 14 & 16 & 16 & 28 & 18 & 19 \\
\hline Gesamt $^{* *}$ & 9 & 25 & 20 & 25 & 43 & 28 & 38 \\
\hline
\end{tabular}

*Die Größenklasseneinteilung erfolgt entlang wichtiger Grenzen laut BetrVG.

**Betriebe der Privatwirtschaft mit mindestens 5 Beschäftigten

Quelle: IAB-Betriebspanel 2014

In Betrieben mit Betriebsrat ist der Anteil von Frauen auf der ersten Führungsebene um fünf Prozentpunkte geringer als in Betrieben ohne Betriebsrat. Dies ist dem mittleren Größensegment zwischen 50 und 500 Beschäftigte geschuldet. Kleinbetriebe und Großbetriebe unterscheiden sich dagegen kaum. Auf der zweiten Führungsebene ist der Unterschied mit 15 Prozentpunkten noch deutlicher. Hier haben Betriebe mit Betriebsrat in allen Größenklassen (deutlich) geringere Anteile von Frauen in Führungspositionen.

Unser spezielles Interesse gilt außerdem Betrieben, in denen Führen im Rahmen einer Teilzeitbeschäftigung möglich ist. Zeigt sich in Betrieben, die solche Möglichkeiten bieten, eine stärkere Präsenz von Frauen in Führungspositionen? Die Ergebnisse in Tabelle 3 zeigen hierzu ein relativ klares Bild.

Tab. 3: Führen in Teilzeit und Frauen in Führungspositionen

\begin{tabular}{|l|c|c|}
\hline \multicolumn{2}{|c|}{ - hochgerechnete Werte - } \\
\hline Anteile in \% & möglich in Teilzeit ist ... \\
\hline Anteil Betriebe & 19 & nicht möglich \\
\hline Frauenanteil im Betrieb & 50 & 41 \\
Frauenanteil auf der ersten Führungsebene & 31 & 23 \\
\hline Frauenanteil auf der zweiten Führungsebene & 52 & 31 \\
\hline
\end{tabular}

Basis: Betriebe der Privatwirtschaft mit mindestens 5 Beschäftigten

Quelle: IAB-Betriebspanel 2014 
Betriebe, die diese Möglichkeit bieten, stehen sowohl auf der ersten als auch und insbesondere auf der zweiten Führungsebene hinsichtlich des Frauenanteils deutlich besser da. Auf der zweiten Führungsebene übertrifft die Präsenz von Frauen in Führungspositionen (52\%) sogar den betrieblichen Frauenanteil (50\%), falls Führen in Teilzeit möglich ist. Immerhin 19 Prozent aller privatwirtschaftlichen Betriebe (ab 5 Beschäftigte) bieten diese Option. Die Anteilswerte steigen mit der Betriebsgröße von 18 Prozent in Kleinbetrieben bis zu 36 Prozent in Großbetrieben (ohne Tabelle). Um festzustellen, inwieweit sich diese deskriptiven Ergebnisse auch unter Berücksichtigung weiterer betrieblicher Merkmale bestätigen, bedarf es einer multivariaten Analyse.

\subsection{Operationalisierung und Variablenbeschreibung}

Für die Modellierung der Präsenz von Frauen in Führungspositionen gibt es keinen einheitlichen oder umfassenden theoretischen Erklärungsansatz, aus dem die Determinanten klar abgeleitet werden könnten. Hinzu kommt, dass die oft festgestellten „unsichtbaren“ Barrieren (glass ceiling) in den Betrieben selbstredend schlecht in Schätzungen modellierbar sind. Deshalb wollen wir im Folgenden versuchen, einen heuristischen Rahmen abzustecken, indem wir soweit möglich aus den verschiedenen theoretischen Erklärungsmodellen und den Ergebnissen empirischer Studien die Auswahl der Variablen und deren erwartete Wirkung ableiten.

Untersuchung zum Glass-Cliff-Ansatz zeigen, dass Frauen eher in Betrieben, die sich in wirtschaftlich schwierigen Situationen befinden, in Führungspositionen gelangen. Deshalb sollen in den Schätzungen einerseits die wirtschaftliche Lage des Betriebs und das Ausmaß des Personalumschlags als Indikatoren für eventuell vorhandene betriebliche Probleme und ungünstige Arbeitsbedingungen aufgenommen werden. Wir gehen davon aus, dass Frauen in Führungspositionen seltener in Betrieben mit einem positiven Jahresergebnis (Dummyvariable) und häufiger in Betrieben mit einem hohen Labour-Turnover (Summe der Zu- und Abgänge/gemittelte Gesamtbeschäftigung für das 1. Halbjahr) zu finden sind.

Die deskriptive Analyse der Daten des IAB-Betriebspanels zeigt, dass Frauen auf der zweiten Führungsebene sehr viel häufiger zu finden sind als auf der ersten. Zumindest deskriptiv zeigen sich also auch mit unseren Daten die „sticky floors“. Da die Doppelbelastung von Frauen und ihre damit verbundene fehlende Flexibilität als Gründe für dieses Phänomen genannt werden, sollen in unseren Schätzungen Variablen berücksichtigt werden, die Frauen bei der Überwindung von Aufstiegsbarrieren helfen sollten. Zunächst ist hier an verschiedene Arbeitszeitregelungen zu denken. Dazu zählt die Nutzung von Arbeitszeitkonten, die gewisse Flexibilitätsspielräume für die Beschäftigten bieten, ebenso wie Vertrauensarbeitszeitregelungen, bei der die Beschäftigten die Arbeits- und Anwesenheitszeiten selbst festlegen und keine betriebsseitige Kontrolle mehr stattfindet. Diese werden als Dummyvariablen zur Arbeitszeitgestaltung in den Modellen berücksichtigt.

Wir erwarten, dass die Möglichkeit, Arbeitszeitkonten zu nutzen, einen positiven Einfluss auf den Anteil von Frauen in Führungspositionen haben sollte. Die zu erwartenden Effekte von Vertrauensarbeitszeitregelungen sind weniger eindeutig. Einerseits könnten entsprechende Angebote die Chancen von Frauen erhöhen, da sie dadurch eher in die Lage versetzt würden, betriebliche und familiäre Erfordernisse zu vereinbaren. Andererseits ist aus Studien zur betrieblichen Praxis von Vertrauensarbeitszeitregelungen bekannt, dass diese vor allem im Bereich hochqualifizierter Tätigkeiten und in Führungsetagen zu einer z.T. deutlichen Verlängerung der Arbeitszeiten führen (Böhm, Herrmann, \& Trinczek, 2004). Möglicher- 
weise ergibt sich durch diese Präsenzkultur ein gegenteiliger Effekt, in dem Frauen (vor dem Hintergrund der ungleichen Lastenverteilung bei der Familienarbeit) eher die Segel streichen bzw. von einem möglichen Schritt ins Management abgehalten werden. Welchen Einfluss Vertrauensarbeitszeit letztlich auf den Anteil der weiblichen Führungskräfte hat, kann damit nur empirisch beantwortet werden.

Von den weiteren personalpolitischen Maßnahmen, die angeboten werden, um betriebliche Aufstiegsbarrieren aufzubrechen, steht uns die Information zur Verfügung, ob Betriebe es ermöglichen, Führungspositionen auch mit reduzierter Stundenzahl auszuüben (Dummyvariable). Da dies vor allem Frauen den Aufstieg in eine Managementposition erleichtern soll, erwarten wir einen positiven Einfluss. Zumal dies auch als ein Signal des Betriebs gesehen werden kann, dass Frauen in Führungspositionen tatsächlich willkommen sind.

Die stärkere Belastung der Frauen mit Familienaufgaben und die damit einhergehende geringere Flexibilität werden auch als eine Erklärung für die niedrigeren Verdienste der Frauen nicht nur in Führungspositionen herangezogen. Demnach haben Frauen oft eine geringere Verhandlungsmacht, weil sie nicht so leicht Optionen außerhalb der eigenen Firma wahrnehmen (können). In den Daten des IAB-Betriebspanels sind allerdings keine Angaben zu den Gehältern der Führungskräfte enthalten, deshalb kann hier nur der durchschnittliche Lohn pro Vollzeitäquivalent als Proxy verwendet werden. Es ist aber davon auszugehen, dass das betriebliche Lohnniveau und die Managergehälter stark korrelieren. Je höher das Lohnniveau im Betrieb, desto geringer wird vermutlich der Anteil der Frauen in Führungspositionen ausfallen.

Die horizontale Segregation der Arbeitsmärkte, wonach Frauen in bestimmten Branchen und Berufen verstärkt zu finden sind, wird in unseren Modellen einerseits durch die Wirtschaftszweigzugehörigkeit operationalisiert. Die Wirtschaftszweigvariablen sollen branchenspezifische Einflüsse aufnehmen, die nicht schon in den übrigen Größen, z.B. etwaige West-Ost-Unterschiede (siehe weiter unten) oder Unterschiede in der Betriebsgröße oder der Beschäftigtenstruktur, Ausdruck finden. Es handelt sich somit um noch nicht adressierte Branchencharakteristika, die den Frauenanteil in Führungspositionen beeinflussen.

Zusätzlich werden der Frauenanteil im Betrieb, die Angabe, ob ein Betrieb gemeinnützig ist und der Anteil der gering- und der einfachqualifizierten Beschäftigten als Proxy-Variablen für die horizontale Segregation in die Schätzungen einbezogen. Hier wird ein positiver Zusammenhang mit dem Anteil von Frauen in Führungspositionen erwartet. Auch der Anteil hochqualifizierter Beschäftigter wird berücksichtigt, allerdings gehen wir davon aus, dass in Betrieben mit einem hohen Anteil Hochqualifizierter der Anteil von Frauen in Führungspositionen eher niedriger ist. Um den Einfluss oder die Machtfülle der Führungskräfte im jeweiligen Betrieb zu erfassen (horizontale Segregation), wird außerdem ein Maß verwendet, das die Leitungsspanne abbilden soll. Es wird berechnet als Quotient aus der Anzahl von Führungskräften und der Anzahl der Beschäftigten, und zwar separat für beide Führungsebenen.

Entscheidungsprozesse in Organisationen hängen in starkem Maß von organisatorischen Regelungen und Strukturen ab. Aus den vorgegebenen Strukturen können sich mehr oder weniger persistente organisationsspezifische „Kulturen“ herausbilden. Überlegungen zur Pfadabhängigkeit (Schreyögg, Koch, \& Sydow, 2004, S. 1301) unterstreichen zusätzlich die Beharrungskraft bestehender betrieblicher Strukturen. Die Entwicklung von Organisationen verläuft demnach entlang bestimmter Pfade, die so leicht nicht verlassen werden. Entsprechend hängen auch aktuelle Entscheidungen von „gewachsenen“ betrieblichen Strukturen ab. Dies kann in männerdominierten Betrieben dazu führen, dass Frauen sehr viel schlechtere 
Chancen haben, in eine Führungsposition zu kommen. Dieses Problem wird besonders in großen und älteren Unternehmen gesehen. Busch und Holst (2012) argumentieren, dass kleinere und jüngere Betriebe weniger bürokratische Strukturen aufweisen und deshalb vermutlich eher in der Lage sind, Frauen in Führungspositionen zu integrieren. Aus diesem Grund wird neben der Betriebsgröße auch das Betriebsalter (als gruppierte Anzahl der Jahre, die die Betriebsgründung zurückliegt) in unseren Analysen berücksichtigt.

$\mathrm{Zu}$ den „gewachsenen“ Strukturen, die vielleicht (noch) einen Einfluss auf die geschlechtsspezifische Zusammensetzung der Führungsebenen haben, gehört auch die Lage des Betriebs in West- oder Ostdeutschland. Auch 25 Jahre nach der Wiedervereinigung zeigen sich in bestimmten Lebensbereichen entsprechende regionale Unterschiede, wie z.B. beim Anteil von Frauen in Führungspositionen, der, wie deskriptive Analysen zeigen, im Osten deutlich höher ausfällt (Kohaut \& Möller, 2016).

Der Effekt der Existenz eines Betriebsrats lässt sich ex ante nicht eindeutig bestimmen. Zunächst könnte natürlich keinerlei Einfluss bestehen - wenn die Interessenvertretung sich diesbezüglich nicht engagieren will. Ein positiver Effekt ist denkbar, wenn der Betriebsrat die ihm allgemein zugewiesene Aufgabe, Chancengleichheit zu fördern und Diskriminierung zu verhindern, ernst nimmt und auch ohne darüber hinaus gehende rechtliche Grundlagen ein (geschlechtssensitives) Auge auf die Rekrutierung und Förderung des Führungspersonals hat. Angesichts der rechtlichen Situation erwarten wir hier eher für die zweite Managementebene einen Einfluss. Andererseits ist vorstellbar, dass männlich dominierte Betriebsratsgremien als Teil der (männlich dominierten) Austauschbeziehungen eher zur Aufrechterhaltung entsprechender Geschlechterstereotypen mit ihren Auswirkungen auf die Besetzung von Führungspositionen (mit einem Bias hin zu männlichen Bewerbern) beitragen.

Über die bisher präsentierten Merkmale hinaus werden einige Kontrollvariablen in die Modelle einbezogen. Als zweite Institution der Arbeitsbeziehung findet die Tarifbindung der Betriebe Berücksichtigung. Tarifverträge beeinflussen die Arbeitsbedingungen maßgeblich. Aus empirischen Studien ist z.B. bekannt, dass der Gender Pay Gap in tarifgebundenen Betrieben niedriger ausfällt als in nicht tarifgebundenen (Grimm, Lang, \& Stephan, 2016). Es ist somit durchaus vorstellbar, dass eine tarifvertragliche Bindung auch Auswirkungen auf die Präsenz von Frauen in Führungspositionen hat. Des Weiteren wird berücksichtigt, ob sich der Betrieb in ausländischem Mehrheitseigentum befindet, um eventuelle Unterschiede in der Organisationskultur ausländischer Betriebe im Vergleich zu deutschen abbilden zu können. Zusätzlich wird dafür kontrolliert, ob tätige Inhaber im Betrieb arbeiten, es sich um ein unabhängiges Unternehmen handelt und der Betrieb einer Handwerkskammer angehört. Diese Variablen charakterisieren jeweils unterschiedliche betriebliche (Entscheidungs-)Strukturen, die möglicherweise auch auf den Anteil von Frauen in Führungspositionen Auswirkungen haben. $^{2}$

\subsection{Schätzstrategie}

In den verfügbaren Daten stehen Informationen zur ersten und zweiten Führungsebene zur Verfügung, so dass wir für beide Ebenen die geschlechtsspezifische Zusammensetzung der Führungsteams betrachten können. Bei der Analyse der ersten Führungsebene schließen wir allerdings Betriebe mit nur einer Führungsposition aus. Häufig handelt es sich hier um Betriebe, die vom Eigentümer oder der Gründerperson geleitet werden. Damit spielen andere

2 Die Mittelwerte der in der Schätzung verwendeten Variablen sind im Anhang Tabelle A1 dargestellt. 
Entscheidungsprozesse bei der Besetzung der obersten Managementposition eine Rolle als in Betrieben mit einem Führungsteam auf der obersten Ebene. Darüber hinaus werden in theoretischen Ansätzen und empirischen Studien zu Frauen in Führungspositionen jeweils Karrierewege betrachtet. In Betrieben mit nur einer Führungsposition an der Spitze stehen eher Besonderheiten bei der Gründung oder die Unternehmensnachfolge in der Familie im Fokus. In unseren Analysen für die zweite Führungsebene werden hingegen alle Betriebe, die eine solche haben, berücksichtigt.

Die uns interessierenden Variablen - die Anzahl der Frauen auf der ersten und zweiten Führungsebene - beinhalten nichtnegative, ganzzahlige Werte, die eine Verteilung aufweisen, für deren Analyse Standard OLS- Regressionsmodelle nicht angemessen sind. Hierzu eigenen sich Zähldatenmodelle mit der Poissonregression als Basisvariante (Hilbe, 2007). Die alternative Betrachtung von Anteilswerten (Anteil weiblicher Führungskräfte an allen Führungskräften) als abhängige Variable ist nicht nur deshalb problematisch, weil sie die zugrundeliegende Verteilungsannahme verletzt. Da solche Anteile sowohl durch eine Veränderung des Zählers als auch des Nenners bestimmt werden, gestaltet sich auch die Interpretation der Ergebnisse schwierig.

Bei der Nutzung von Zähldatenmodellen sollte zunächst überprüft werden, ob Überdispersion (die bedingten Varianzen sind höher als die bedingten Mittelwerte) vorliegt (was zu inkonsistenten Koeffizienten führen würde). Die entsprechenden statistischen Tests geben aber keinen Hinweis auf solche Probleme.

Zum anderen sollte beim Vorliegen einer größeren Anzahl von Nullwerten in der abhängigen Variablen (keine Frau in der jeweiligen Führungsetage) entschieden werden, ob es eine zusätzliche systematische Quelle von Nullen gibt (excess oder certain zeros), die in einem weiteren datengenerierenden Prozess erzeugt werden. In einem solchen Fall müsste auf entsprechende „Zero-Inflated“ Regressionsmodelle zurückgegriffen werden, in denen neben dem Zähldatenmodell ein separates „Inflationsmodell“ für das (sichere) Auftreten von Nullen geschätzt wird. Wir gehen davon aus, dass es bei unserem Gegenstand keine solchen zusätzlichen (sicheren) Nullwerte gibt, die in einer eigenen Schätzung berücksichtigt werden müssten (siehe zur Diskussion um die Notwendigkeit von Zero-Inflated-Models: Allison, 2012).

Damit die Ergebnisse des Zähldatenmodells dennoch im Sinne einer Quote interpretiert werden können und die Anzahl der Frauen in einer Führungsposition mit der Gesamtzahl der Führungspositionen einen Bezugspunkt hat, muss die logarithmierte Anzahl der Führungspositionen als zusätzliche technische Variable in die Schätzung einbezogen werden.

Vor dem Hintergrund, dass die Variablen zur wirtschaftlichen Situation des Betriebs und vor allem zum Lohnniveau einen nicht unerheblichen Anteil fehlender Werte aufweisen (über 800 Fälle bei den Schätzungen zur ersten und mehr als 1300 Fälle bei Schätzungen zur zweiten Führungsebene), haben wir als Robustheitscheck Modelle auch mit einem entsprechend reduzierten Variablenset und deutlich höheren Fallzahlen gerechnet (siehe Tabelle A2 im Anhang). Die Ergebnisse unterscheiden sich nur insoweit als einige Variablen (bei vergleichbaren Koeffizienten) in den Schätzungen mit den höheren Fallzahlen die Signifikanzgrenze überschreiten. 


\subsection{Ergebnisse}

$\mathrm{Ob}$ und in welcher Form die oben vorgestellten Variablen tatsächlich einen Einfluss auf die Präsenz von Frauen in Führungspositionen haben, ist in Tabelle 4 dargestellt. Es werden die exponenzierten Koeffizienten ( $\left.\exp ^{b}\right)$ ausgewiesen, die interpretierbar sind als der Effekt einer Veränderung der unabhängigen Variablen um eine Einheit auf die Rate der abhängigen Variable (incidence rate). Werte größer 1 bedeuten einen positiven, kleiner 1 einen negativen Einfluss. Ein Wert von 0,95 bedeutet z.B. eine Reduzierung der Rate um 5 Prozent.

Zunächst sollen die Ergebnisse des Modells für die erste Führungsebene näher betrachtet werden. Die verwendeten Indikatoren für eine wirtschaftlich schwierige Situation des Betriebs zeigen den erwarteten Einfluss. So sind in Betrieben mit einem negativen Jahresergebnis häufiger Frauen in Führungspositionen zu finden als in solchen mit positivem oder ausgeglichenem. Für die zweite Determinante, den Personalumschlag, gilt, je höher der Labour-Turnover im Betrieb, desto häufiger sind Frauen an der Spitze.

Die Indikatoren für eine flexible Arbeitszeitgestaltung haben unterschiedliche Auswirkungen auf den Anteil von Frauen in Führungspositionen. Vorhandene Arbeitszeitkonten haben zwar das erwartete Vorzeichen, allerdings ist der Effekt nicht signifikant. Eine betriebliche Vertrauensarbeitszeitregelung geht hingegen mit einer geringeren Wahrscheinlichkeit einher, dass Frauen Positionen auf der ersten Führungsebene bekleiden. Anscheinend überwiegt hier der aus anderen Studien bekannte Effekt, dass Vertrauensarbeitszeit eher zu überlangen Arbeitszeiten führt, auch wenn die Lage und Verteilung der Arbeitszeit frei gewählt werden kann.

Das Angebot, die Führungsposition auch im Rahmen einer Teilzeitbeschäftigung ausüben zu können, erhöht wie erwartet den Frauenanteil dagegen deutlich. Damit scheint diese Maßnahme, die explizit zur Förderung von Frauen angeboten wird, die intendierte Wirkung zu entfalten. Wie Eingangs schon erwähnt, verbinden wir aber mit unseren Erläuterungen keinen kausalen Erklärungsanspruch. Grundsätzlich könnte hier - wie auch bei vielen anderen Merkmalen - ein gegenteiliger Zusammenhang bestehen. Wenn (mehr) Frauen Führungspositionen bekleiden, sorgen sie dafür, dass es solche Angebote gibt, um anderen Kandidatinnen (trotz Doppelbelastung) den Aufstieg zu ermöglichen.

Die betrachtete Lohnvariable zeigt den erwarteten (signifikant) negativen Effekt. Je höher das Lohnniveau im Betrieb ist, desto seltener findet man Frauen in Toppositionen. Die geringere Flexibilität und damit verbunden die geringere Verhandlungsmacht der Frauen spielt hier vermutlich ebenso eine Rolle wie die Tatsache, dass Frauen eher in Berufen arbeiten, die schlechter bezahlt werden. 
Tab. 4: Poisson-Reggression zur Anzahl Frauen in Führungspositionen 2014

Basis: privatwirtschaftliche Betriebe ab 5 Beschäftigte

\begin{tabular}{|c|c|c|c|c|c|c|}
\hline & \multicolumn{3}{|c|}{$\begin{array}{l}\text { 1. Führungsebene } \\
\text { (Führungsteams) }\end{array}$} & \multicolumn{3}{|c|}{ 2. Führungsebene } \\
\hline & $\exp (b)$ & & Std.f. & $\exp (b)$ & & Std.f. \\
\hline Positives Jahresergebnis (ja=1) & 0,854 & -- & $(0,044)$ & 0,966 & & $(0,030)$ \\
\hline Labour-Turnover (Zu- u. Abgänge/Personalbestand) & 1,748 & +++ & $(0,204)$ & 1,266 & +++ & $(0,107)$ \\
\hline Arbeitszeitkonten $(j a=1)$ & 1,047 & & $(0,056)$ & 0,946 & - & $(0,028)$ \\
\hline Vertrauensarbeitszeit (ja=1) & 0,902 & -- & $(0,045)$ & 0,966 & & $(0,028)$ \\
\hline Teilzeit für Führungspersonal (ja=1) & 1,112 & ++ & $(0,060)$ & 1,290 & +++ & $(0,039)$ \\
\hline Lohn pro Vollzeitäquivalent & 0,871 & -- & $(0,049)$ & 0,803 & --- & $(0,032)$ \\
\hline Frauenanteil & 4,776 & +++ & $(0,534)$ & 7,100 & +++ & $(0,502)$ \\
\hline Gemeinnütziger Betrieb (ja=1) & 1,205 & + & $(0,119)$ & 1,022 & & $(0,053)$ \\
\hline Anteil Einfachqualifikationen & 1,113 & & $(0,131)$ & 1,089 & & $(0,066)$ \\
\hline Anteil geringfügig Beschäftigte & 0,971 & & $(0,154)$ & 0,985 & & $(0,097)$ \\
\hline Anteil Hochqualifizierter & 0,550 & --- & $(0,105)$ & 0,949 & & $(0,097)$ \\
\hline Beschäftigtenzahl (log) & 0,939 & - & $(0,035)$ & 0,926 & --- & $(0,011)$ \\
\hline Leitungsspanne (Führungskräfte/Mitarbeiter) & 1,412 & & $(0,566)$ & 1,871 & ++ & $(0,473)$ \\
\hline Betrieb 7 bis 12 Jahre alt (ja=1) & 0,915 & & $(0,080)$ & 1,037 & & $(0,062)$ \\
\hline Betrieb 13 bis 18 Jahre alt (ja=1) & 0,988 & & $(0,089)$ & 1,167 & +++ & $(0,063)$ \\
\hline Betrieb 19 bis 24 Jahre alt (ja=1) & 1,012 & & $(0,087)$ & 1,095 & + & $(0,054)$ \\
\hline $\begin{array}{l}\text { Betrieb mehr als } 24 \text { Jahre alt (ja=1) } \\
\text { (Referenz: Betrieb bis } 6 \text { Jahre alt) }\end{array}$ & 1,025 & & $(0,078)$ & 1,077 & & $(0,055)$ \\
\hline Standort in Westdeutschland (ja=1) & 0,765 & -- & $(0,043)$ & 0,825 & --- & $(0,030)$ \\
\hline Betriebsrat $(j a=1)$ & 0,899 & & $(0,066)$ & 0,958 & & $(0,034)$ \\
\hline Tarifvertrag (ja=1) & 1,006 & & $(0,053)$ & 1,035 & & $(0,032)$ \\
\hline Auslandskontrolle (ja=1) & 1,018 & & $(0,119)$ & 0,992 & & $(0,051)$ \\
\hline Tätige Inhaber (ja=1) & 0,998 & & $(0,055)$ & 0,984 & & $(0,032)$ \\
\hline Zweigstelle, Mittelinstanz (ja=1) & 0,986 & & $(0,081)$ & 0,985 & & $(0,039)$ \\
\hline Handwerksbetrieb (ja=1) & 1,187 & +++ & $(0,073)$ & 1,071 & + & $(0,044)$ \\
\hline Konstante & 0,549 & & $(0,273)$ & 0,948 & & $(0,287)$ \\
\hline Dummies für Wirtschaftszweige & & ja & & & ja & \\
\hline Wald $\mathrm{Chi}^{2}$ & & 1024,8 & & & 5965,4 & \\
\hline Pseudo $\mathrm{R}^{2}$ & & 0,14 & & & 0,28 & \\
\hline Fallzahl (Betriebe) & & 2962 & & & 4275 & \\
\hline
\end{tabular}

Anmerkung: +++/++/+ bzW. ---/--/- signalisieren einen signifikant positiven bzw. negativen Zusammenhang auf dem 1\%-/5\%-/10\%-Niveau.

Quelle: IAB-Betriebspanel 2014, eigene Schätzungen 
Die verschiedenen Indikatoren einer horizontalen Segregation haben alle die erwarteten Vorzeichen. So sind Frauen in Managementpositionen häufiger anzutreffen in Betrieben mit einem hohen Frauenanteil und einem höheren Anteil an Gering- und Einfachqualifizierten, wobei die beiden letzten Determinanten nicht signifikant sind. Umgekehrt sinkt mit steigendem Anteil von hochqualifizierten Mitarbeitern im Betrieb der Anteil weiblicher Führungskräfte signifikant. Darüber hinaus haben Frauen in als gemeinnützig anerkannten Betrieben bessere Chancen auf eine Spitzenposition.

Es lassen sich auch branchenspezifische Unterschiede erkennen: Die geringsten Chancen, angemessen in den Führungsetagen vertreten zu sein, haben Frauen in den Finanz- und Versicherungsdienstleistungen, im Baugewerbe, im Bereich Bergbau, Energie, Wasserversorgung, im verarbeitenden Gewerbe und im Handel. Darin zeigen sich möglicherweise organisationskulturelle Aspekte, tradierte Werte und Pfadabhängigkeiten, die besonders entlang von Branchengrenzen auftreten (hier wären sicherlich das Baugewerbe, das Verarbeitende Gewerbe und der Bereich Bergbau, Energie, Wasserversorgung zu nennen). Oder es handelt sich um männlich dominierte Karrieremuster in einer besonderen branchenspezifischen Ausprägung (hierbei wäre an die Finanz- und Versicherungsdienstleistungen zu denken).

Das negative Vorzeichen der Leitungsspanne geht in die erwartete Richtung und deutet darauf hin, dass Frauen eher in eine Führungsposition gelangen, wenn weniger Beschäftigte der Führungskraft unterstellt sind. Allerdings bleibt das Ergebnis insignifikant.

Die Betriebsgröße hat den vermuteten (allerdings nur schwach signifikant) negativen Einfluss auf die Präsenz von Frauen auf der obersten Führungsebene. Hingegen zeigt sich für das Betriebsalter kein signifikanter Einfluss und die Koeffizienten widersprechen tendenziell der Vermutung, dass Frauen eher in jüngeren Betrieben in eine Führungsposition gelangen.

Als weitere Variable sei die regionale Lage des Betriebs erwähnt, die einen starken Einfluss auf die Präsenz von Frauen hat. In Ostdeutschland stehen die Chancen für Frauen deutlich besser als im Westen. Ob hier das unterschiedliche Erwerbsverhalten von Frauen in der ehemaligen DDR Nachwirkungen zeigt, muss offen bleiben. Zumal auch dort Frauen offenbar nicht angemessen in Führungspositionen vertreten waren (Hofmann, 2000). Die Vermutung liegt nahe, dass tradierte Rollenverständnisse wohl eher in Westdeutschland nach wie vor den Aufstieg von Frauen behindern.

Wie sieht es nun mit dem Betriebsrat aus? Der Koeffizient für die Existenz eines Betriebsrats weist auf der ersten Führungsetage in Richtung einer Reduzierung der Chancen von Frauen, bleibt aber insignifikant. ${ }^{3}$ Dieses Ergebnis spricht gegen einen Betriebsrat, der sich die Förderung der Chancengleichheit und die Verhinderung von Diskriminierung zur Aufgabe gemacht hat. Eher lassen sich die Ergebnisse in Richtung eines Betriebsrats interpretieren, der als Teil der traditionellen Organisationskultur mit zur Benachteiligung von Frauen beiträgt.

Die berücksichtigten Kontrollvariablen zeigen überwiegend keinen Einfluss auf den Anteil von Frauen in Führungspositionen. Das gilt auch für die Tarifbindung. Nur in Handwerksbetrieben haben Frauen bessere Chancen auf eine Führungsposition. Eine mögliche Erklärung hierfür könnte sein, dass es sich dabei oft um Familienbetriebe handelt, die von (Ehe-)Partnern oder mehreren Familienangehörigen arbeitsteilig geführt werden. Es wäre dann nicht unplausibel, dass sich ein vergleichsweise hoher Frauenanteil bei solchen in der

3 Bei der Schätzung ohne die Lohnvariable zeigt sich hier ein schwach signifikant negativer Zusammenhang. 
Regel kleinen Führungsteams ergibt. (Zur Erinnerung: Betriebe mit nur einer Führungsposition sind bei den Schätzungen für die erste Führungsebene ausgeschlossen.)

Verglichen mit der Schätzung für die erste Ebene weist das Modell für Frauen auf der zweiten Führungsebene eine deutlich bessere Modellanpassung auf. Hinsichtlich der Einflussfaktoren zeigt sich eine große Übereinstimmung, weshalb wir uns im Folgenden auf die abweichenden Ergebnisse beschränken. Während der Labour-Turnover gleichermaßen einen hoch signifikant positiven Einfluss auf den Anteil von Frauen auf der zweiten Führungsebene hat, verliert die wirtschaftliche Lage, gemessen als positives Jahresergebnis, ihre Signifikanz. Das verwundert jedoch nicht, da sich die Glass-Cliff-Hypothese (operationalisiert durch die wirtschaftliche Lage) vor allem auf die oberste Ebene bezieht.

Überraschend sind die Ergebnisse für die Arbeitszeitvariablen. Im Gegensatz zur ersten Führungsebene hat das Vorhandensein von Arbeitszeitkonten hier einen negativen und signifikanten Einfluss auf die Präsenz von Frauen in Führungspositionen. Eine Erklärung hierfür könnte sein, dass Frauen sehr viel häufiger in Betrieben arbeiten (und entsprechend auch in Führungspositionen zu finden sind), in denen in Schicht oder anderen stark reglementierten bzw. getakteten Arbeitszeitregimes gearbeitet wird. Ein Schichtbetrieb wie er in Heimen, Krankenhäusern oder auch im Handel vorkommt, setzt einer flexiblen Arbeitszeitgestaltung mit Hilfe von Arbeitszeitkonten enge Grenzen.

Die Vertrauensarbeitszeit wird zwar mit der gleichen Wirkrichtung wie auf der ersten Ebene geschätzt. Allerdings ist der Einfluss nicht mehr signifikant. Das mag daran liegen, dass überlange Arbeitszeiten auf der zweiten Führungsebene weit weniger zum Führungsstil gehören als im Topmanagement.

Bei der Betriebsgröße zeigt sich der erwartete (negative) Zusammenhang auf der zweiten Führungsebene deutlicher. Die Aufstiegschancen von Frauen reduzieren sich insbesondere im mittleren Management mit zunehmender Betriebsgröße. Der in der Literatur gleichermaBen vermutete Einfluss des Betriebsalters - Frauen schaffen es eher in jungen Betrieben in eine Führungsposition - wird aber auch für die zweite Führungsebene nicht bestätigt. Hier zeigt sich vielmehr, dass vor allem in Betrieben mittleren Alters (13-23 Jahre) Frauen signifikant häufiger in Führungspositionen gelangen.

Die Variable zur Gemeinnützigkeit eines Betriebes behält zwar ihr positives Vorzeichen, allerdings ist der Effekt nicht mehr signifikant. Im Gegensatz dazu wird der Einfluss der Leitungsspanne hoch signifikant. Das bedeutet, dass vor allem auf der zweiten Führungsebene der Frauenanteil umgekehrt proportional zur Anzahl der geführten Personen ist, Frauen also in diesem Sinne in ihren Führungspositionen über eine geringere Machtfülle verfügen als Männer.

Obwohl wir auf der zweiten Führungsebene eher einen Einfluss des Betriebsrats auf die Präsenz von Frauen im Management erwartet hatten, bleibt der Koeffizient insignifikant mit negativem Vorzeichen. ${ }^{4}$ Das Ergebnis deutet auch für das mittlere Management darauf hin, dass der Betriebsrat sein Augenmerk eher nicht auf die Chancengleichheit von Männer und Frauen legt. 


\section{Fazit}

Ziel dieses Beitrags war es zu analysieren, welche betrieblichen Charakteristika die Präsenz von Frauen in Führungspositionen beeinflussen, und zwar auf den beiden höchsten Managementebenen. In unseren Schätzungen haben wir verschiedene Merkmale betrachtet, die in den einschlägigen theoretischen und empirischen Arbeiten zu geschlechtsspezifischen Karrieremechanismen adressiert werden und einer Umsetzung und Überprüfung mit Betriebsdaten zugänglich sind. Als wesentlicher Bestandteil der Organisationskultur bzw. der Arbeitsbeziehungen wird die betriebliche Interessenvertretung in den Modellen mitberücksichtigt.

Die Schätzergebnisse für beide Führungsebenen stimmen weitgehend überein. Insbesondere lassen sich aber für die erste Führungsebene Hinweise darauf finden, dass es in den Betrieben wohl tatsächlich so etwas wie ,gläserne Klippen“ gibt und Böden bzw. Etagen, auf denen Frauen „kleben bleiben“. Insgesamt vermitteln die Ergebnisse aber vor allem den Eindruck, dass Frauen in ,unattraktiven“ Betrieben bessere Chancen auf eine Führungsposition haben. Diese Betriebe schreiben eher rote Zahlen, haben einen höheren Personalumschlag, bezahlen niedrigere Löhne, haben ein geringeres Qualifikationsniveau und die Führungspositionen verfügen über eine geringere Machtfülle (geringere Leitungsspanne).

Unsere Modelle liefern auch Hinweise auf die Wirksamkeit personalpolitischer Maßnahmen. So erhöht die Möglichkeit, eine Führungsposition im Rahmen einer Teilzeitbeschäftigung zu bekleiden, die Chancen von Frauen deutlich.

Hinsichtlich der Rolle des Betriebsrats bei der geschlechtsspezifischen Besetzung von Führungspositionen hatten wir alternative Hypothesen formuliert, je nachdem ob davon ausgegangen wird, dass dieser seine vorhandenen Machtressourcen für die Durchsetzung einer emanzipatorischen Besetzungs- und Förderpolitik einsetzt oder eher traditionelle Organisationsstrukturen für ein „,business as usual“" sorgen, bei dem Frauen nach wie vor eher geringe Chancen auf eine Führungsposition haben. Unsere Ergebnisse weisen eher auf letzteres hin, auch wenn die Ergebnisse in unserem vollständigen Modell nicht signifikant sind. Allerdings ist der Betriebsrat nur als Dummy-Variable in den Schätzungen berücksichtigt. Diese eher monolithische Betrachtung des Betriebsrats führt möglicherweise dazu, dass vorhandene, gegenläufige Zusammenhänge überdeckt werden. Hier bedarf es weiterer Forschung mit einer differenzierteren Betrachtung des Betriebsrats z.B. hinsichtlich seiner geschlechtsspezifischen Zusammensetzung (insbesondere des Betriebsratsvorsitzes) oder hinsichtlich des Alters des Betriebsratsgremiums und seines organisationskulturellen Status.

In gewerkschaftlichen Kampagnen und entsprechenden Verlautbarungen wird zwar auf die mögliche Vorbildfunktion des Betriebsrats für die Frauenförderung (sprich: mehr Frauen als Betriebsratsvorsitzende) und der Rolle, die ein „emanzipiertes“ Gremium für eine geschlechtssensible betriebliche Besetzungs- und Förderungspraxis spielen könnte, hingewiesen (z.B. IG Metall, 2016; Homann-Kramer \& Krug 2014). Allerdings scheinen diese Bemühungen bislang weder auf die Frauenquote beim Betriebsratsvorsitz noch auf die in den Führungsetagen der Betriebe mit Betriebsrat einen spürbaren Einfluss zu haben.

Sowohl hinter der Besetzung von Führungspositionen als auch dem individuellen Aufstieg in Führungspositionen steht eine Vielzahl von Faktoren, die unsere empirischen Analysen nur zum Teil abdecken können. So werden individuelle Motive und Eigenschaften in den betrieblichen Modellen natürlich gar nicht abgebildet. Aber auch innerhalb der Betriebe gibt es Einflussgrößen, die sich quantitativ nur schwer oder gar nicht erfassen lassen. Die bessere Güte des Modells für die zweite Führungsebene gibt einen Hinweis darauf, dass die erklären- 
den Variablen besser geeignet sind, die Präsenz von Frauen auf der zweiten Führungsebene zu erklären. Offensichtlich spielen insbesondere für die Besetzung von Führungspositionen auf der ersten Ebene andere, teilweise nicht quantifizierbare Faktoren eine Rolle. Dazu gehören z.B. Informationen über die Bedeutung informeller Netzwerke oder die Zusammensetzung und Entscheidungsprozesse der Gremien bzw. Zirkel, die für die Besetzung von Toppositionen letztlich verantwortlich sind. Nicht von ungefähr wird in der einschlägigen Literatur auch von unsichtbaren Barrieren gesprochen.

\section{Literatur}

Allison, P. (2012). Do We Really Need Zero-Inflated Models? Abgerufen am 10.02.2016 von http:// statisticalhorizons.com/zero-inflated-models - https://doi.org/10.1007/978-3-322-99707-4_12

Allmendinger, J., \& Hinz, T. (1999). Geschlechtersegregation im Erwerbsbereich. Berufsfelder, Organisationen, Arbeitsgruppen. In W. Glatzer \& I. Ostner (Hrsg.), Deutschland im Wandel-Sozialstrukturelle Analysen (S. 191-205). Opladen.https://doi.org/10.1007/978-3-322-99707-4_12

Barreto, M., \& Ellemers, N. (2005). The burden of benevolent sexism: How it contributes to the maintenance of gender inequalities. European Journal of Social Psychology, 35, 633-642. doi 10.1002/ ejsp. 270

Baumann, H., \& Brehmer, W. (2016). Die Zusammensetzung von Betriebsräten: Ergebnisse aus der WSI-Betriebsrätebefragung 2015. WSI-Mitteilungen, 69, 201-210.

Billing, Y.D. \& Alvesson, M. (2014). Leadership: A Matter of Gender? In S. Kumra, R. Simpson, \& R.J. Burge (Hrsg.), The Oxford Handbook of Gender in Organizations (S. 200-222). Oxford University Press.

Böhm, S., Herrmann, C., \& Trinczek, R. (2004). Herausforderung Vertrauensarbeitszeit: Zur Kultur und Praxis eines neuen Arbeitszeitmodells. Forschung aus der Hans-Böckler-Stiftung, 54, Berlin.

Booth, A.L., Francesconi, M., \& Frank, J. (2003). A sticky floors model of promotion, pay, and gender. European Economic Review, 47, 295-322. https://doi.org/10.1016/S0014-2921(01)00197-0

Bosch, A., Ellguth, P., Schmidt, R., \& Trinczek, R. (1999). Betriebliches Interessenhandeln. 1. Opladen. doi 10.1007/978-3-663-09391-6

Busch, A., \& Holst, E. (2009). Berufswahl wichtig für Karrierechancen von Männern und Frauen. DIW Wochenbericht, 23, 376-384.

Busch, A., \& Holst, E. (2010). Der Gender Pay Gap in Führungspositionen: Warum die Humankapitaltheorie zu kurz greift. Femina Politica, 19, 91-101.

Busch, A., \& Holst, E. (2012). Occupational Sex Segregation and Management-Level Wages in Germany: What Role Does Firm Size Play? IZA Discussion Paper 6568.

Calas, M.B., Smircich, L., \& Holvino, E. (2014). Theorizing Gender-and-Organization: Changing Times...Changing Theories. In S. Kumra, R. Simpson, R., \& R.J. Burge (Hrsg.), The Oxford Handbook of Gender in Organizations (S. 17-52). Oxford University Press.

Cotter, D.A., Hermsen, J.M., Ovadia, S., \& Vanneman, R. (2001). The Glass Ceiling Effect. Social Forces, 80, 655-682. https://doi.org/10.1353/sof.2001.0091

Die bayerischen Metall-und Elektro-Arbeitgeber (2016). Frauen in Führungspositionen - Staffel III Abgerufen am 06.12.2016 von www.baymevbm.de/Frauen

Dilger, A. (2002). Ökonomik betrieblicher Mitbestimmung. Die wirtschaftlichen Folgen von Betriebsräten. München.

Ellguth, P., Kohaut, S., \& Möller, I. (2014). The IAB Establishment Panel - Methodological Essentials and Data Quality. Journal for Labour Market Research, 47, 27-41. doi 10.1007/s12651-013-0151-0

Ellguth, P., \& Kohaut, S. (2016). Tarifbindung und betriebliche Interessenvertretung: Ergebnisse aus dem IAB-Betriebspanel 2015. WSI-Mitteilungen, 69, 283-291.

Fietze, S., Holst, E., \& Tobsch, V. (2011). Germany's Next Top Manager: Does Personality Explain the Gender Career Gap? Management Revue, 22, 240-273. 
Fischer, G., Janik, F., Müller, D., \& Schmucker, A. (2008). The IAB Establishment Panel - Things Users Should Know. In: Schmollers Jahrbuch (Journal of Applied Social Science Studies), 129, 133-148.

Graham, M. E., Belliveau, M. A., \& Hotchkiss, J. L. (2016). The View at the Top or Signing at the Bottom? Workplace Diversity Responsibility and Women's Representation in Management. ILR Review, 20, 1-36.

Greifenstein, R., Kißler, L., \& Lange, H. (2014). Trendreport Betriebsrätewahlen 2014 - Zwischenbericht. Marburg.

Grimm, V., Lang, J., \& Stephan, G. (2016). Tarifverträge und die Lohnlücke zwischen Männern und Frauen: Empirische Evidenz aus Zerlegungsanalysen. Industrielle Beziehungen, 23, 309-333.

Hilbe, J.M. (2007). Negative Binomial Regression. Cambridge. doi 10.1017/cbo9780511811852

Hofmann, L.M. (2000): Führungskräfte in Europa: Empirische Analyse zukünftiger Anforderungen. Wiesbaden.

Homann-Kramer, K., \& Krug, G.(2014). Betriebsrätinnen als Vorbild. Arbeitsrecht im Betrieb, 10, 45-47.

Holst, E. (2009). Führungskräftemonitor 2001-2006. Forschungsreihe des Bundesministeriums für Familie, Senioren, Frauen und Jugend, Band 7. Baden-Baden.

Holst, E., \& Friedrich, M. (2016). Hohe Führungspositionen: In der Finanzbranche haben Frauen im Vergleich zu Männern besonders geringe Chancen. DIW Wochenbericht, 37, 827-839.

Holst, E., \& Kirsch, A. (2016). Managerinnen-Barometer 2016. DIW Wochenbericht, 2, 46-57.

Holst, E., \& Wiemer, A. (2010). Frauen sind in Spitzengremien der Wirtschaft unterrepräsentiert. Wirtschaftsdienst, 10, 692-699. doi 10.1007/s10273-010-1137-y

IG Metall (2016). Abgerufen am 06.12.2016 von http://www.wer-die-besten-will.de/materialien/

Jackson J. F.L., \& O'Callaghan E. M. (2009). What do we know about glass ceiling effects? A taxonomy and critical review to inform higher education research. Research in Higher Education, 50, 460-482. doi 10.1007/s11162-009-9128-9

Jirjahn, U. (2011). Gender, Worker Representation and the Profitability of Firms in Germany. The European Journal of Comparative Economics, 8, 281-298.

Joecks, J., Pull, K., \& Vetter, K. (2012). Gender Diversity in the Boardroom and Firm Performance: What Exactly Constitutes a „Critical Mass“? Journal of Business Ethics, 118, 61-72. https://doi.org/10.1007/s10551-012-1553-6

Klempt, C., \& Klee, G. (2015). Frauen in Führungspositionen 2014. Empirische Analyse auf der Basis des IAB-Betriebspanels Baden-Württemberg. IAW Kurzberichte, 3.

Kohaut, S., \& Möller, I. (2016). Im Osten sind Frauen öfter an der Spitze. IAB-Kurzbericht, 2.

Krishnan, H. (2009). What causes turnover among women on top management teams? Journal of Business Research, 62, 1181-1186. doi 10.1016/j.jbusres.2008.09.001

Metz, I., \& Kulik, C.T. (2014). The Rocky Climb: Women's Advancement in Management. In S. Kumra, R. Simpson, \& R.J. Burge (Hrsg.): The Oxford Handbook of Gender in Organizations (S. 175199). Oxford University Press.

Orser, B., \& Leck, J. (2010). Gender influence on career success outcomes. Gender in Management: An International Journal, 25, 386-407. doi 10.1108/17542411011056877

Powell, G.N. (2014). Sex, Gender, and Leadership: What Do Four Decades of Research Tell Us? In S. Kumra, R. Simpson, \& R.J. Burge (Hrsg.): The Oxford Handbook of Gender in Organizations (S. 249-268). Oxford University Press.

Schreyögg, G., Koch, J., \& Sydow, J. (2004). Routinen und Pfadabhängigkeit. In G. Schreyögg, \& von Werder (Hrgs.), Handwörterbuch Unternehmensführung (S. 1295-1304). Stuttgart.

Schulz, F., \& Blossfeld, H.-P. (2006). Wie verändert sich die häusliche Arbeitsteilung im Eheverlauf? Eine Längsschnittstudie der ersten 14 Ehejahre in Westdeutschland. Kölner Zeitschrift für Soziologie und Sozialpsychologie, 58, 23-49. doi 10.1007/s11575-006-0002-0

Smith, N., Smith, V., \& Verner, M. (2013). Why Are So Few Females Promoted into CEO and Vice President Positons? Danish Empirical Evidence, 1997-2007. ILRReview, 66, 380-408.

Stettes, O. (2015). Betriebsratswahlen 2014 - eine Rückblick auf Basis der Betriebsratswahlbefragung, IW-Trend, 1. 


\section{Anhang}

Tab. A1: Mittelwerte der verwendeten Variablen

\begin{tabular}{|c|c|c|}
\hline & $\begin{array}{l}\text { 1. Führungsebene } \\
\text { (Führungsteams) }\end{array}$ & 2. Führungsebene \\
\hline Variablen & \multicolumn{2}{|c|}{ Mittelwerte (Stichprobenwerte) } \\
\hline Anteil Frauen auf der Führungsebene & 0,21 & 0,33 \\
\hline Anzahl Frauen auf der Führungsebene & 0,58 & 1,32 \\
\hline Betrieb macht Gewinn & 0,77 & 0,77 \\
\hline Labour-Turnover & 0,11 & 0,12 \\
\hline Arbeitszeitkonten & 0,64 & 0,66 \\
\hline Vertrauensarbeitszeit & 0,38 & 0,38 \\
\hline Teilzeit für Führungspersonal & 0,22 & 0,23 \\
\hline Lohn pro Vollzeitäquivalent (log) & 2.642 & 2.647 \\
\hline Frauenanteil & 0,37 & 0,38 \\
\hline Gemeinnütziger Betrieb & 0,05 & 0,05 \\
\hline Anteil Einfachqualifikationen & 0,17 & 0,19 \\
\hline Anteil geringfügig Beschäftigte & 0,09 & 0,09 \\
\hline Anteil Hochqualifizierter & 0,10 & 0,10 \\
\hline Beschäftigtenzahl (log) & 225 & 191 \\
\hline Leitungsspanne (Anzahl FK/Anzahl Besch.) & 0,09 & 0,08 \\
\hline Betrieb 7 bis 12 Jahre alt & 0,11 & 0,12 \\
\hline Betrieb 13bis 18 Jahre alt & 0,11 & 0,11 \\
\hline Betrieb 19 bis 24 Jahre alt & 0,21 & 0,24 \\
\hline Betrieb mehr als 24 Jahre alt & 0,47 & 0,43 \\
\hline Standort in Westdeutschland & 0,68 & 0,63 \\
\hline Betriebsrat (ja=1) & 0,31 & 0,35 \\
\hline Tarifvertrag & 0,41 & 0,44 \\
\hline Auslandskontrolle & 0,07 & 0,09 \\
\hline Tätige Inhaber & 0,53 & 0,47 \\
\hline Zweigstelle, Mittelinstanz & 0,11 & 0,18 \\
\hline Handwerksbetrieb & 0,25 & 0,23 \\
\hline Fallzahl & 2.962 & 4.275 \\
\hline
\end{tabular}

Quelle: IAB-Betriebspanel 2014, Betriebe der Privatwirtschaft ab 5 Beschäftigte 
A2: Poisson-Reggression zur Anzahl Frauen in Führungspositionen 2014

Basis: privatwirtschaftliche Betriebe ab 5 Beschäftigte

\begin{tabular}{|c|c|c|c|c|c|c|}
\hline & \multicolumn{3}{|c|}{$\begin{array}{l}\text { 1. Führungsebene } \\
\text { (Führungsteams) }\end{array}$} & \multicolumn{3}{|c|}{ 2. Führungsebene } \\
\hline & $\exp (b)$ & & Std.f. & $\exp (b)$ & & Std.f. \\
\hline Labour-Turnover (Zu- u. Abgänge/Personalbestand) & 1,699 & +++ & $(0,150)$ & 1,313 & +++ & $(0,072)$ \\
\hline Arbeitszeitkonten (ja=1) & 1,055 & & $(0,046)$ & 0,963 & & $(0,023)$ \\
\hline Vertrauensarbeitszeit (ja=1) & 0,878 & --- & $(0,036)$ & 0,991 & & $(0,024)$ \\
\hline Teilzeit für Führungspersonal (ja=1) & 1,123 & +++ & $(0,049)$ & 1,231 & +++ & $(0,030)$ \\
\hline Frauenanteil & 4,813 & +++ & $(0,446)$ & 7,014 & +++ & $(0,440)$ \\
\hline Gemeinnütziger Betrieb (ja=1) & 1,260 & +++ & $(0,088)$ & 1,035 & & $(0,039)$ \\
\hline Anteil Einfachqualifikationen & 1,248 & ++ & $(0,121)$ & 1,128 & ++ & $(0,057)$ \\
\hline Anteil geringfügig Beschäftigte & 1,028 & & $(0,141)$ & 1,138 & & $(0,090)$ \\
\hline Anteil Hochqualifizierter & 0,672 & --- & $(0,085)$ & 0,865 & -- & $(0,059)$ \\
\hline Beschäftigtenzahl (log) & 0,941 & -- & $(0,027)$ & 0,912 & --- & $(0,009)$ \\
\hline Leitungsspanne (Führungskräfte/Mitarbeiter) & 1,990 & ++ & $(0,613)$ & 1,470 & ++ & $(0,273)$ \\
\hline Betrieb 7 bis 12 Jahre alt (ja=1) & 1,057 & & $(0,080)$ & 1,018 & & $(0,049)$ \\
\hline Betrieb 13 bis 18 Jahre alt (ja=1) & 1,053 & & $(0,083)$ & 1,141 & +++ & $(0,052)$ \\
\hline Betrieb 19 bis 24Jahre alt (ja=1) & 1,022 & & $(0,076)$ & 1,059 & & $(0,043)$ \\
\hline $\begin{array}{l}\text { Betrieb mehr als } 24 \text { Jahre alt (ja=1) } \\
\text { (Referenz: Betrieb bis } 6 \text { Jahre alt) }\end{array}$ & 1,040 & & $(0,069)$ & 1,045 & & $(0,043)$ \\
\hline Standort in Westdeutschland $(j a=1)$ & 0,756 & -- & $(0,034)$ & 0,791 & -- & $(0,022)$ \\
\hline Betriebsrat (ja=1) & 0,900 & - & $(0,051)$ & 0,956 & - & $(0,027)$ \\
\hline Tarifvertrag (ja=1) & 1,009 & & $(0,043)$ & 1,059 & ++ & $(0,025)$ \\
\hline Auslandskontrolle (ja=1) & 0,908 & & $(0,088)$ & 0,978 & & $(0,043)$ \\
\hline Tätige Inhaber (ja=1) & 0,995 & & $(0,046)$ & 1,010 & & $(0,028)$ \\
\hline Zweigstelle, Mittelinstanz (ja=1) & 1,078 & & $(0,068)$ & 1,002 & & $(0,031)$ \\
\hline Handwerksbetrieb (ja=1) & 1,177 & +++ & $(0,063)$ & 1,102 & +++ & $(0,041)$ \\
\hline Konstante & 0,158 & & $(0,026)$ & 0,200 & & $(0,018)$ \\
\hline Dummies für Wirtschaftszweige & & ja & & & ja & \\
\hline Wald $\mathrm{Chi}^{2}$ & & 1500 & & & 8124 & \\
\hline Pseudo $\mathrm{R}^{2}$ & & 0,15 & & & 0,29 & \\
\hline Fallzahl (Betriebe) & & 3811 & & & 5631 & \\
\hline
\end{tabular}

Anmerkung: +++/++/+ bzw. ---/--/- signalisieren einen signifikant positiven bzw. negativen Zusammenhang auf dem 1\%-/5\%-/10\%-Niveau.

Quelle: IAB-Betriebspanel 2014, eigene Schätzungen 


\section{DuEPublico}

Dieser Text wird über DuEPublico, dem Dokumenten- und Publikationsserver der Universität Duisburg-Essen, zur Verfügung gestellt. Die hier veröffentlichte Version der EPublikation kann von einer eventuell ebenfalls veröffentlichten Verlagsversion abweichen.

DOI: $\quad$ 10.3224/indbez.v24i2.05

URN: urn:nbn:de:hbz:464-20200716-155244-6 